\title{
FOURIER-DELIGNE TRANSFORM AND REPRESENTATIONS OF THE SYMMETRIC GROUP
}

\author{
Galyna Dobrovolska
}

\begin{abstract}
We calculate the Fourier-Deligne transform of the IC extension to $\mathbb{C}^{n+1}$ of the local system $\mathcal{L}_{\Lambda}$ on the cone over $\operatorname{Conf}_{n}\left(\mathbb{P}^{1}\right)$ associated with a representation $\Lambda$ of the symmetric group $S_{n}$, where the length $n-k$ of the first row of the Young diagram of $\Lambda$ is at least $\frac{|\Lambda|-1}{2}$. The answer is the IC extension to the dual vector space $\mathbb{C}^{n+1}$ of the local system $\mathcal{R}_{\lambda}$ on the cone over the $k$ th secant variety of the rational normal curve in $\mathbb{P}^{n}$, where $\mathcal{R}_{\lambda}$ corresponds to the representation $\lambda$ of $S_{k}$, the Young diagram of which is obtained from the Young diagram of $\Lambda$ by deleting its first row. We also prove an analogous statement for $S_{n}$-local systems on fibers of the Abel-Jacobi map. We use our result on the Fourier-Deligne transform to rederive a part of a result of Michel Brion on Kronecker coefficients.
\end{abstract}

\section{Introduction}

Let $W \cong \mathbb{C}^{2}$ be a two-dimensional complex vector space. For any positive integer $r$ we can identify the projective space $\mathbb{P}^{r}=\mathbb{P}\left(\operatorname{Sym}^{r}(W)\right)$ with degree $r$ polynomials in two variables up to scaling, and consider the discriminant hypersurface $D_{r}$ in $\mathbb{P}^{r}$ which is formed by polynomials with multiple roots. The complement $\mathbb{P}^{r}-D_{r}$ is identified with the configuration space $\operatorname{Conf}_{r}\left(\mathbb{P}^{1}\right)$ of $r$ unordered distinct points on the projective line $\mathbb{P}^{1}$. Hence, $\pi_{1}\left(\mathbb{P}^{r}-D_{r}\right)$ is the spherical braid group $B_{r}\left(\mathbb{P}^{1}\right)$. It is known that $B_{r}\left(\mathbb{P}^{1}\right)=B_{r} /\left(b_{1} b_{2} \cdots b_{r-1} b_{r-1} \cdots b_{2} b_{1}\right)$, where $b_{1}, b_{2}, \ldots, b_{r-1}$ are the standard generators of the braid group $B_{r}$, and the usual map $B_{r} \rightarrow S_{r}$ to the symmetric group $S_{r}$ on $r$ letters factors through $B_{r}\left(\mathbb{P}^{1}\right)$. Hence a representation $\rho$ of $S_{r}$ gives a representation of $B_{r}\left(\mathbb{P}^{1}\right)$ and thus a local system $\mathcal{L}_{\rho}$ on $\mathbb{P}^{r}-D_{r}$. We denote by $\mathbf{L}_{\rho}$ the perverse sheaf on the complex vector space $\operatorname{Sym}^{r}(W)$ which is the intermediate extension to $\operatorname{Sym}^{r}(W)$ of the lift of $\mathcal{L}_{\rho}$ to the complement of the cone over $D_{r}$ in $\operatorname{Sym}^{r}(W)$. The goal of this paper is to find the Fourier-Deligne transform (see Definition 2.1 below or Chapter III of $[\mathrm{KS}]$ ) of such objects $\mathbf{L}_{\rho}$.

The Fourier-Deligne transform for similar local systems on the set of semisimple regular matrices is important in Springer theory, see [B], Section XI, or [KW], Chapter VI, and for similar local systems on curves of higher genus it is important in the geometric Langlands program in the paper $[\mathrm{D}]$ of Drinfeld.

The main result of this paper is Theorem 1.1 in which we calculate the FourierDeligne transform of $\mathbf{L}_{\rho}$ where the first row of $\rho$ has length at least $\frac{|\rho|-1}{2}$ (in this case we say that $\rho$ has a long first row); Theorem 1.1 is generalized to the case of an arbitrary smooth curve embedded into projective space in Theorem 1.4. Theorem 1.1 is also used to derive Proposition 1.5 in which we find a relation with Michel Brion's result in [Brion], Corrollary 2, Section 3.4.

Received by the editors January 26, 2013. 
1.1. Main theorem for $\mathbb{P}^{\mathbf{1}}$. For a Young diagram denoted by a capital Greek letter, we denote the Young diagram obtained by deleting its first row by the corresponding lowercase Greek letter. (Note that we use the same notation for a representation of $S_{r}$ and for its Young diagram.) Let $|\Lambda|=n$ and let $k=|\lambda|$. Consider the rational normal curve $X$ in the projective space $\mathbb{P}\left(\operatorname{Sym}^{n}\left(W^{*}\right)\right)$, which is the dual variety of the hypersurface $D_{n} \subset \mathbb{P}\left(\operatorname{Sym}^{n}(W)\right)$. The $k$ th secant variety $\operatorname{Sec}^{k}=\operatorname{Sec}^{k}(X)$ of $X$ is the closure of the union of " $k$-secant" $(k-1)$-planes $\mathbb{P}^{k-1}$ that pass through $k$ distinct points of $X$. When $k \leq \frac{n+1}{2}$, (i.e., $\Lambda$ has a long first row) the variety $\mathrm{Sec}^{k}-\mathrm{Sec}^{k-1}$ is smooth and has the structure of a fiber bundle over the unordered configuration space $\mathbb{P}^{k}-D_{k} \cong \operatorname{Conf}_{k}\left(\mathbb{P}^{1}\right)$ (see Lemma 3.5 ); the cone $U_{k}$ (with vertex removed) over $\mathrm{Sec}^{k}-\mathrm{Sec}^{k-1}$ in $\operatorname{Sym}^{n}\left(W^{*}\right)$ shares the same properties. We define a local system $\mathcal{R}_{\lambda}$ on $U_{k}$ by pulling back the local system $\mathcal{L}_{\lambda}$ from $\mathbb{P}^{k}-D_{k}$ via the bundle map. We denote the perverse sheaf, which is the intermediate extension of $\mathcal{R}_{\lambda}$ to $\operatorname{Sym}^{n}\left(W^{*}\right)$ by $\mathbf{R}_{\lambda}$.

Theorem 1.1. If $\Lambda$ has a long first row, the Fourier-Deligne transform of the perverse sheaf $\mathbf{L}_{\Lambda}$ on the vector space $\operatorname{Sym}^{n}(W)$ is the perverse sheaf $\mathbf{R}_{\lambda}$ on $\operatorname{Sym}^{n}\left(W^{*}\right)$.

Example 1.2. Let $\Lambda$ be the standard representation of $S_{n}$. In this case we can verify Theorem 1.1 using the facts that (1) the Fourier transform $\Phi$ agrees with the Radon transform $R$ up to constant complexes, and (2) the Radon transform of a complex $\mathcal{F}$ on a subvariety $Y$ in $\mathbb{P}^{n}$ is given by monodromy of hyperplane sections of the cone over $Y$ with coefficients in the lift of $\mathcal{F}$ (see [B]).

Take $Y=X$, the rational normal curve in $\mathbb{P}\left(V^{*}\right)$, and the constant sheaf on $X$. A generic hyperplane section of the cone over $X$ in $V^{*}$ is isomorphic to $\mathbb{P}^{1}$ punctured at $n$ points, and a basis of $H^{1}$ of this hyperplane section is formed by small circles around the punctures. It is clear with this basis that monodromy of hyperplane sections yields the defining representation of $S_{n}$, (i.e., $\Lambda \oplus$ triv). Since the trivial representation gives us a constant sheaf, we see that (up to constant complexes) the Radon transform $R$ sends $\mathbf{R}_{\lambda}$ to $\mathbf{L}_{\Lambda}$ (note that $\operatorname{Sec}^{1}(X)=X, \lambda$ is the trivial representation of $S_{1}$, and $\mathcal{R}_{\lambda}$ is the trivial local system). Using involutivity and the fact that $\Phi$ sends irreducible perverse sheaves to irreducible ones, we obtain $\Phi\left(\mathbf{L}_{\Lambda}\right)=\mathbf{R}_{\lambda}$.

Example 1.3. Note that for Theorem 1.1 to hold the Young diagram $\Lambda$ must have a long first row. Indeed, consider the simplest Young diagram $\Lambda$ which does not have a long first row, namely the Young diagram for the sign representation of $S_{4}$. For this representation Theorem 1.1 is false: in fact, in this case $\Phi\left(\mathbf{L}_{\Lambda}\right)$ is a perverse sheaf corresponding to a local system on an open subset of $\operatorname{Sym}^{n}\left(W^{*}\right)$ such that the corresponding monodromy representation does not factor through a finite group.

To see this, note that a general fiber of the map $\pi^{\perp}: \mathcal{E}^{\perp} \rightarrow \operatorname{Sym}^{4}\left(W^{*}\right)$ (used in the proof of Theorem 1.1) is a copy of $\mathbb{P}^{1}$. In the course of proving Theorem 1.1, we show that $\Phi\left(\mathbf{L}_{\Lambda}\right)$ is a direct summand in the direct image under $\pi^{\perp}$ of the perverse sheaf on the total space of $\mathcal{E}^{\perp}$, such that its restriction to a general fiber of $\pi^{\perp}$ is the IC extension of the local system on $\mathbb{P}^{1}$ minus four points corresponding to the sign representation of $S_{3}$. (Indeed, by construction of the bundle $\mathcal{E}^{\perp}$ and the sheaf on it, a generic fiber of the map $\pi^{\perp}$ is a projective line in the base $\mathbb{P}^{3}$ of the bundle $\mathcal{E}^{\perp}$; the local system on the open subset of the above $\mathbb{P}^{1}$ is obtained by restricting the sign local system on the complement of the discriminant hypersurface in $\mathbb{P}^{3}$.) 
We conclude that the monodromy of the local system in the summand $\Phi\left(\mathbf{L}_{\Lambda}\right)$ of the direct image under $\pi^{\perp}$ is the Picard-Lefschetz monodromy of a family of elliptic curves, which is infinite. (Note that we get elliptic curves because the cohomology of $\mathbb{P}^{1}$ with coefficients in the IC extension of the sign local system on the complement of four points is a summand in the cohomology of the elliptic curve which is the double cover of this $\mathbb{P}^{1}$ ramified at these four points.)

1.2. Main theorem for any curve. Let $C$ be a smooth projective curve of genus $g$. Let $C_{\text {dist }}^{(n)}$ be the open subvariety of $C^{(n)}$ (the symmetric power of $C$ ) consisting of the $n$-tuples of distinct points of $C$. For a representation $\Lambda$ of $S_{n}$, consider the local system $\mathcal{L}_{\Lambda}^{C \text {,big }}$ on $C_{\text {dist }}^{(n)}$ corresponding to the representation of $\pi_{1}\left(C_{\text {dist }}^{(n)}\right)$ which is the pullback of $\Lambda$ via the natural map $\pi_{1}\left(C_{\text {dist }}^{(n)}\right) \rightarrow S_{n}$. Let $n>2 g-2$ so that the classical Abel-Jacobi map $C^{(n)} \rightarrow \operatorname{Pic}^{n}(C)$ is a fibration.

Restricting the local system $\mathcal{L}_{\Lambda}^{C \text {,big }}$ to a fiber of the Abel-Jacobi map over a degree $n$ line bundle $M$ on $C$, we obtain a local system $\mathcal{L}_{\Lambda}^{C}$ on an open subset of the vector space $\mathbb{P}\left(H^{0}(M)\right)$. From this local system we construct a perverse sheaf $\mathbf{L}_{\Lambda}^{C}$ on the vector space $H^{0}(M)$ as before by taking the intermediate extension of the lift of the local system $\mathcal{L}_{\Lambda}^{C}$ to the cone. Denote by $\operatorname{Sec}^{k}(C)$ the secant variety of $C$ embedded into $\mathbb{P}\left(H^{0}(M)^{*}\right)$, and by $\mathbf{R}_{\lambda}^{C}$ the perverse sheaf supported on the cone over $\operatorname{Sec}^{k}(C)$ constructed as before by intermediate extension of the lift of $\mathcal{L}_{\lambda}^{C \text {, big }}$ via the bundle map (see Lemma 4.3(c)). Let $\lambda$ still denote the partition obtained from $\Lambda$ by deleting its first row. We have a result similar to Theorem 1.1:

Theorem 1.4. If $|\lambda| \leq \frac{n+1}{2}-g$, then

$$
\Phi\left(\mathbf{L}_{\Lambda}^{C}\right)=\mathbf{R}_{\lambda}^{C} .
$$

1.3. Relation with $M$. Brion's result. Let $\Lambda$ and $\Omega$ be two Young diagrams with $|\Lambda|=|\Omega|=n,|\lambda| \leq \frac{n+1}{2}$ and $|\omega| \leq \frac{n+1}{2}$. (For a partition denoted by a capital Greek letter, we still denote the partition obtained by deleting its first row by the corresponding lowercase Greek letter.) Recall that for a representation $\Sigma$ of $S_{n}$ we have the Kronecker coefficient $k_{\Lambda, \Omega}^{\Sigma}=[\Sigma: \Lambda \otimes \Omega]$; if $|\sigma|=|\lambda|+|\omega|$ we can also define the Littlewood-Richardson coefficient $c_{\lambda, \omega}^{\sigma}=\left[\sigma: \operatorname{Ind}_{S_{|\lambda|} \times S_{|\omega|}}^{S_{|\sigma|}} \lambda \otimes \omega\right]$. We prove:

Proposition 1.5. Suppose that $|\lambda|+|\omega| \leq n / 2$. Then

(a) For any Young diagram $\Sigma$ with the first row strictly shorter than $n-|\lambda|-|\omega|$, the Kronecker coefficient $m_{\Lambda, \Omega}^{\Sigma}$ is zero.

(b) For any Young diagram $\Sigma$ with the first row equal to $n-|\lambda|-|\omega|$, the Kronecker coefficient $k_{\Lambda, \Omega}^{\Sigma}$ is equal to the Littlewood-Richardson coefficient $c_{\lambda, \omega}^{\sigma}$.

Remark 1.6. This result is the same as Corollary 2 in Section 3.4 of [Brion] except for our additional assumption $|\lambda|+|\omega| \leq n / 2$. Without this assumption our method encounters some technical difficulties.

1.4. Organization of the paper. Section 2 is devoted to background on the FourierDeligne transform. In Section 3 we prove a number of lemmas about the FourierDeligne transform and apply them to prove Theorem 1.1. In Section 4 we generalize Theorem 1.1 to local systems on fibers of the Abel-Jacobi map. In Section 5, we deduce from Theorem 1.1 a part of M. Brion's result as Proposition 1.5. 


\section{Fourier-Deligne transform}

The general reference for this section is the book [KS]; see also the book [KW] and the survey article [I], in which the theory is described in the $\ell$-adic setting, and the papers [B, BMV1, BMV2], in which the Fourier-Deligne transform is studied. Let $Y$ be an algebraic variety over $\mathbb{C}$ (or a real analytic stratified space). We work with the derived category of constructible sheaves $D_{c}^{b}(Y)$. For a morphism of complex algebraic varieties (or stratified spaces) $f: Y \rightarrow Z$ we have the functors $f_{*}, f_{!}: D_{c}^{b}(Y) \rightarrow D_{c}^{b}(Z)$ and $f^{*}, f^{!}: D_{c}^{b}(Z) \rightarrow D_{c}^{b}(Y)$ (note that we write $f_{*}$ for $R f_{*}$ and similarly for the other functors; we also use $\otimes$ in place of $\left.\otimes^{L}\right)$.

Perverse sheaves $\operatorname{Perv}(Y)$ form an abelian subcategory of $D_{c}^{b}(Y)$. The classification theorem of $[\mathrm{BBD}]$ states that every irreducible perverse sheaf on $Y$ is obtained by extending to $Y$ by zero an object of the form $\operatorname{IC}(\bar{U}, \mathcal{L})$, where $\mathcal{L}$ is an irreducible local system on a smooth locally closed subvariety $U \subset Y$. Here $\operatorname{IC}(\bar{U}, \mathcal{L})$ stands for the IC extension of $\mathcal{L}$ to the closure of $U$ (also referred to as Goresky-MacPherson or intermediate extension). We also recall that the category of local systems on a manifold $U$ is equivalent to the category of representations of $\pi_{1}(U)$.

Definition 2.1. Let $V$ be a vector space over $\mathbb{C}$ and $p_{1}: V \times V^{*} \rightarrow V, p_{2}: V \times$ $V^{*} \rightarrow V^{*}$ be the projections. Let $j: Q=\{(v, \xi) \mid \operatorname{Re}\langle v, \xi\rangle \leq 0\} \hookrightarrow V \times V^{*}$ and $\delta_{Q}=j_{*}(\underline{\mathbb{C}})$. The Fourier-Deligne transform $\Phi: D_{c}^{b}(V) \rightarrow D_{c}^{b}\left(V^{*}\right)$ is defined by $\Phi(\mathcal{F})=\left(\mathrm{p}_{2}\right) !\left(\mathrm{p}_{1}^{*}(\mathcal{F}) \otimes \delta_{\mathrm{Q}}\right)$.

Note that sometimes we refer to $\Phi$ simply as "the Fourier transform."

Remark 2.2. (a) $\Phi$ is defined using a stratification of $V \times V^{*}$ which is only real analytic, but if $\mathcal{F}$ is constructible with respect to a complex analytic stratification on $V, \Phi(\mathcal{F})$ is likewise on $V^{*}$.

(b) $\Phi$ sends the category $D_{c, \text { mon }}^{b}(V)$ of monodromic objects on $V$ to $D_{c \text {, mon }}^{b}\left(V^{*}\right)$; in this text we always work with $\Phi: D_{c, \text { mon }}^{b}(V) \rightarrow D_{c, \text { mon }}^{b}\left(V^{*}\right)$.

(c) $\Phi$ sends perverse sheaves to perverse sheaves and induces an exact functor between the abelian categories of perverse sheaves on $V$ and $V^{*}$.

Remark 2.3. $\Phi$ can be defined for $\ell$-adic sheaves on $V$ over $\mathbb{F}_{q}$; then it is compatible (under the Frobenius trace) with the Fourier transform for functions on $V$ (see $[\mathrm{I}]$ ).

Remark 2.4. The Weyl algebra $\mathfrak{W}$ is the free algebra $\mathbb{C}\left\langle x_{0}, \ldots x_{n}, \partial_{0}, \ldots, \partial_{n}\right\rangle$ modulo the relations $x_{i} \partial_{j}=\partial_{j} x_{i}$ for $i \neq j$ and $\partial_{i} x_{i}-x_{i} \partial_{i}=1$. The regular holonomic Dmodules $\mathrm{D}-\bmod ^{\text {r.h. }}(V)$ on $V$ form a subcategory of the category of modules over $\mathfrak{W}$. The Riemann-Hilbert correspondence RH: $D-\bmod ^{r . h \cdot}(V) \rightarrow \operatorname{Perv}(V)$ is an equivalence of categories. Under RH the Fourier-Deligne transform defined above is compatible with the Fourier transform $F$ for $\mathfrak{W}$-modules given as follows. If $M$ is a $\mathfrak{W}$-module, $F(M)=M$ as a vector space and the new action of $\mathfrak{W}$ on $m \in M$ is $x_{i}^{\text {new }}(m)=\partial_{i}(m)$, $\partial_{i}^{\text {new }}(m)=-x_{i}(m)$.

In the course of proving, our main result we also need the following more general definition of the (relative) Fourier transform:

Definition 2.5. Let $E$ be a complex vector bundle over a complex algebraic variety $Y, E^{*}$ the dual bundle, and $p_{1}: E \times_{Y} E^{*} \rightarrow E, p_{2}: E \times_{Y} E^{*} \rightarrow E^{*}$ be the projections. 
Let $j: Q=\{((x, v),(x, \xi)) \mid \operatorname{Re}\langle v, \xi\rangle \leq 0\} \hookrightarrow E \times_{Y} E^{*}$ and $\delta_{Q}=j_{*}(\underline{\mathbb{C}})$. The FourierDeligne transform $\Phi_{E}: D_{c}^{b}(E) \rightarrow D_{c}^{b}\left(E^{*}\right)$ is defined by $\Phi_{\mathrm{E}}(\mathcal{F})=\left(\mathrm{p}_{2}\right) !\left(\mathrm{p}_{1}^{*}(\mathcal{F}) \otimes \delta_{\mathrm{Q}}\right)$.

We note that the remarks above apply here, and we will work with $\Phi_{E}: D_{c, \text { mon }}^{b}(E)$ $\rightarrow D_{c, \text { mon }}^{b}\left(E^{*}\right)$, where $\mathbb{C}^{*}$ acts in the fibers of $E$ and $E^{*}$.

\section{Proof of the main theorem for $\mathbb{P}^{1}$}

Let $W \cong \mathbb{C}^{2}$ be a two-dimensional complex vector space. Consider the bilinear pairing $\omega: \operatorname{Sym}^{k}(W) \times \operatorname{Sym}^{n-k}(W) \rightarrow \operatorname{Sym}^{n}(W)$ which corresponds to multiplication of polynomials. The linear maps $\omega(v,-)$, obtained by fixing the first coordinate in the pairing $\omega$ to be $v$, combine for all $v$ to give a map of vector bundles over $\mathbb{P}^{k}=$ $\mathbb{P}\left(\operatorname{Sym}^{k}(W)\right)$, namely $i: \mathcal{E}=\mathcal{O}_{\mathbb{P}^{k}}(-1) \otimes \operatorname{Sym}^{n-k}(W) \rightarrow \mathcal{O}_{\mathbb{P}^{k}} \otimes \operatorname{Sym}^{n}(W)$. We denote the bundle map for $\mathcal{E}$ by $p_{\mathcal{E}}: \mathcal{E} \rightarrow \mathbb{P}^{k}$. Note that the map $i$ makes $\mathcal{E}$ a sub-bundle of the trivial bundle $\mathcal{O}_{\mathbb{P}^{k}} \otimes \operatorname{Sym}^{n}(W)$, so the total space of $\mathcal{E}$ embeds into $\mathbb{P}^{k} \times \operatorname{Sym}^{n}(W)$. Hence, via the second projection we get a map $\pi: \mathcal{E} \rightarrow \operatorname{Sym}^{n}(W), \pi=p_{2} \circ i$. (Note that we sometimes use the same notation for a vector bundle and its total space as we did for $\mathcal{E}$.) See the diagram below.

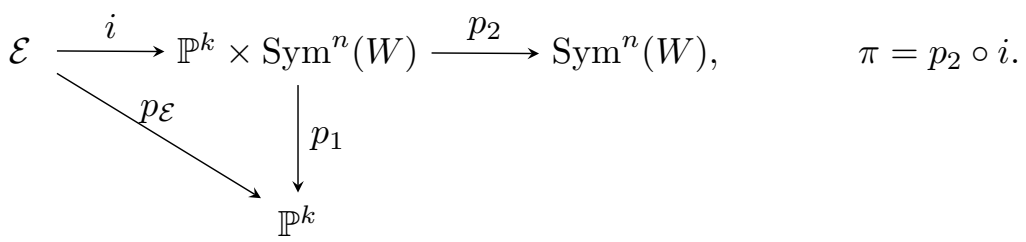

Recall that for a positive integer $r$ and a representation $\rho$ of $S_{r}$ we introduced the local system $\mathcal{L}_{\rho}$ on the open set $\mathbb{P}^{r}-D_{r}$ in $\mathbb{P}^{r}=\mathbb{P}\left(\operatorname{Sym}^{r}(W)\right)$ where $D_{r}$ is the discriminant locus. We denote by $\widetilde{L}_{\rho}$ the intermediate extension of $\mathcal{L}_{\rho}$ to $\mathbb{P}^{r}$ and by $\mathbf{L}_{\rho}$ the intermediate extension to $\operatorname{Sym}^{r}(W)$ of the lift of $\mathcal{L}_{\rho}$ to the complement of the cone over $D_{r}$ in $\operatorname{Sym}^{r}(W)$.

Lemma 3.1. Let $\lambda$ be a representation of $S_{k}$ and let $I$ denote the trivial representation of $S_{n-k}$. Consider the induced representation of $S_{n}$ given by $\bar{\Lambda}=\operatorname{Ind}_{S_{k} \times S_{n-k}}^{S_{n}}(\lambda \otimes I)$. Then, with the above notation, we have $\mathbf{L}_{\bar{\Lambda}}=\pi_{*} p_{\mathcal{E}}^{*} \widetilde{L}_{\lambda}[n-k]$.

Proof. For any positive integer $d$ consider the map $\phi_{d}:\left(\mathbb{P}^{1}\right)^{d} \rightarrow\left(\mathbb{P}^{1}\right)^{(d)}=\mathbb{P}^{d}$. Note that for any representation $\rho$ of $S_{d}$, we have that $\widetilde{L}_{\rho}=\operatorname{Hom}_{S_{d}}\left(\rho,\left(\phi_{d}\right)_{*} \underline{\mathbb{C}}[d]\right)$ (if $\rho$ is an irreducible representation then $\widetilde{L}_{\rho}$ is the isotypic component for the action of $S_{d}$ on the sheaf $\left.\left(\phi_{d}\right)_{*} \underline{\mathbb{C}}[d]\right)$. Also let $m=m_{k, n-k}: \mathbb{P}^{k} \times \mathbb{P}^{n-k} \rightarrow \mathbb{P}^{n}$ denote the map given by multiplication of polynomials.

Note that $\phi=\phi_{n}$ factors through $\mathbb{P}^{k} \times \mathbb{P}^{n-k}$ as $\phi=m \circ\left(\phi_{k} \times \phi_{n-k}\right)$, as depicted in the following diagram:

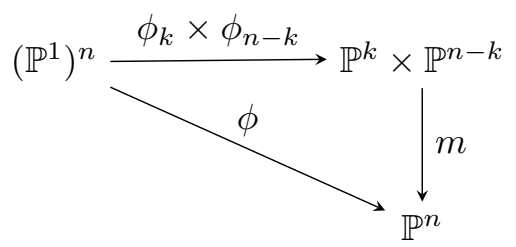


Further, for any representations $\mu$ of $S_{k}$ and $\nu$ of $S_{n-k}$ we have $\widetilde{L}_{\mu} \otimes \widetilde{L}_{\nu}=$ $\operatorname{Hom}_{\mathrm{S}_{\mathrm{k}} \times \mathrm{S}_{\mathrm{n}-\mathrm{k}}}\left(\mu \otimes \nu,\left(\phi_{\mathrm{k}} \times \phi_{\mathrm{n}-\mathrm{k}}\right) * \mathbb{C}[\mathrm{n}]\right)$ on $\mathbb{P}^{k} \times \mathbb{P}^{n-k}$.

We have the following consequence of these observations:

$$
\begin{aligned}
m_{*}\left(\widetilde{L}_{\mu} \otimes \widetilde{L}_{\nu}\right) & =m_{*} \operatorname{Hom}_{\mathrm{S}_{\mathrm{k}} \times \mathrm{S}_{\mathrm{n}-\mathrm{k}}}\left(\mu \otimes \nu,\left(\phi_{\mathrm{k}} \times \phi_{\mathrm{n}-\mathrm{k}}\right)_{*} \underline{\mathbb{C}}[\mathrm{n}]\right) \\
& =\operatorname{Hom}_{\mathrm{S}_{\mathrm{k}} \times \mathrm{S}_{\mathrm{n}-\mathrm{k}}}\left(\mu \otimes \nu, \phi_{*} \underline{\mathbb{C}}[\mathrm{n}]\right)=\operatorname{Hom}_{\mathrm{S}_{\mathrm{n}}}\left(\operatorname{Ind}_{\mathrm{S}_{\mathrm{k}} \times \mathrm{S}_{\mathrm{n}-\mathrm{k}}}^{\mathrm{S}_{\mathrm{n}}}(\mu \otimes \nu), \phi_{*} \underline{\mathbb{C}}[\mathrm{n}]\right) .
\end{aligned}
$$

Note that when we delete the zero section of the bundle $\mathcal{E}$ and take the quotient by the $\mathbb{C}^{*}$-action, (i.e., pass to the projectivization of the vector bundle $\mathcal{E}$ ), the map $\pi$ is replaced by the map $m$ above. Hence we obtain the statement of the lemma from the above chain of equalities by taking $\mu=\lambda$ and $\nu=I$.

The following two lemmas on the Fourier transform are proved in [KW] as Corollary III.13.4 and Corollary III.13.3.

Lemma 3.2. Let $L$ be a constructible complex on a complex algebraic variety $A$. Let $F$ be a sub-bundle of the trivial bundle $A \times W$, where $W$ is a vector space, with bundle map $p_{F}: F \rightarrow A$ and inclusion $i: F \rightarrow A \times W$. Let $F^{\perp}$ be the orthogonal sub-bundle of $A \times W^{*}$, with bundle map $p_{F^{\perp}}: F^{\perp} \rightarrow A$ and inclusion $i^{\perp}: F^{\perp} \rightarrow A \times W^{*}$. Then

$$
\Phi_{A \times W}\left(i_{*} p_{F}^{*} L\right)=i_{*}^{\perp} p_{F^{\perp}}^{*} L\left[\operatorname{dim}\left(F^{\perp}\right)-\operatorname{dim}(F)\right] .
$$

Lemma 3.3. Let $q: S \rightarrow B$ be a proper map of complex algebraic varieties, let $F$ be a vector bundle on $B$, let $q^{*} F$ be the pullback bundle on $S$, and $Q: q^{*} F \rightarrow F$ the map of total spaces of bundles. Let $R: q^{*} F^{*} \rightarrow F^{*}$ be the corresponding map for the total space of the dual bundle. Then for a monodromic constructible complex $M$ on the bundle $q^{*} F$ we have

$$
\Phi_{F}\left(Q_{*} M\right)=R_{*}\left(\Phi_{q^{*} F} M\right) .
$$

We let $\mathcal{E}^{\perp}$ be the sub-bundle of the trivial bundle $\mathbb{P}^{k} \times \operatorname{Sym}^{n}\left(W^{*}\right)$ over $\mathbb{P}^{k}$ which is orthogonal to the sub-bundle $\mathcal{E}$ of $\mathbb{P}^{k} \times \operatorname{Sym}^{n}(W)$. Let $p_{\mathcal{E}^{\perp}}: \mathcal{E}^{\perp} \rightarrow \mathbb{P}^{k}$ be the bundle map, and let $\pi^{\perp}=p_{2}^{\perp} \circ i^{\perp}$ be the composition of the inclusion $i^{\perp}$ of $\mathcal{E}^{\perp}$ into $\mathbb{P}^{k} \times \operatorname{Sym}^{n}\left(W^{*}\right)$ and the second projection $p_{2}^{\perp}$. See the following diagram.

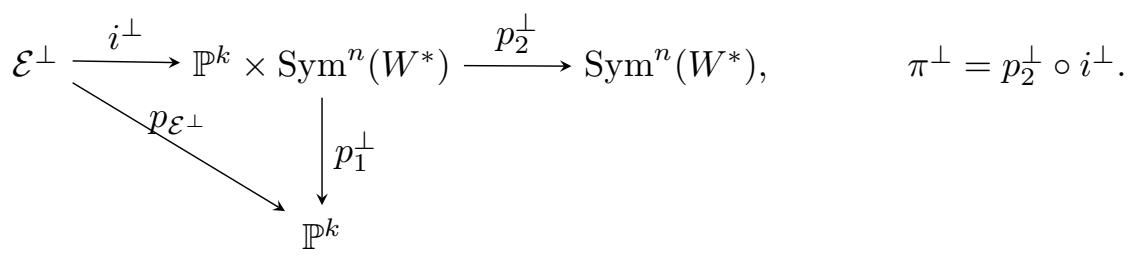

Combining the results of the last three lemmas, we obtain

Proposition 3.4. Using the notation of Lemma 3.1, we have

$$
\Phi\left(\mathbf{L}_{\bar{\Lambda}}\right)=\pi_{*}^{\perp} p_{\mathcal{E}^{\perp}}^{*} \widetilde{L}_{\lambda}[k] .
$$

Proof. Note that we apply Lemma 3.3 to the proper map $\mathbb{P}^{k} \rightarrow$ point.

Lemma 3.5. (a) The image of $\mathcal{E}^{\perp}$ under $\pi^{\perp}$ is the cone over $\operatorname{Sec}^{k}$ in $\operatorname{Sym}^{n}\left(W^{*}\right)$. (b) If $k \leq \frac{n+1}{2}$, the map $\pi^{\perp}$ is an isomorphism restricted to $\left(\pi^{\perp}\right)^{-1}\left(U_{k}\right)$ where $U_{k}$ is the cone (with vertex removed) over $\mathrm{Sec}^{k}-\operatorname{Sec}^{k-1}$ in $\operatorname{Sym}^{n}\left(W^{*}\right)$. 
(c) If $k \leq \frac{n+1}{2}$, the map $p_{\mathcal{E}^{\perp}}$ restricted to $\left(\pi^{\perp}\right)^{-1}\left(U_{k}\right)$ is a fiber bundle over the configuration space $\operatorname{Conf}_{k}(X)=\mathbb{P}^{k}-D_{k}$.

Proof. To prove (a) we note that the fiber of $\mathcal{E}^{\perp}$ over $q \in\left(\mathbb{P}^{1}\right)^{(k)}$ can be thought of as the space of homogeneous polynomials $p$ of degree $n$ which are annihilated by $q\left(\partial_{x}, \partial_{y}\right)$. On the other hand, Lemma 4 in [CS] (see also [L], Section 3.5.3) says that a point $p \in \operatorname{Sym}^{n}\left(W^{*}\right)$ is in $\operatorname{Sec}^{k}$ if and if only there exists a polynomial $q\left(\partial_{x}, \partial_{y}\right)$ which annihilates $p(x, y)$. (Note that Lemma 4 is stated in [CS] only for $k \leq \frac{n}{2}$ but for $k=\frac{n+1}{2}$, if it is an integer, the statement of Lemma 4 in [CS] holds trivially.)

Parts (b) and (c) follow from the fact that (for $k \leq \frac{n+1}{2}$ ) two different $k$-secant planes of $X$ meet at points of the $(k-1)$ th secant variety, (i.e., there is only one $k$-secant plane that passes through a point of $\mathrm{Sec}^{k}-\mathrm{Sec}^{k-1}$ ), which follows by calculating a Vandermonde determinant. A more general version of this argument appears in the proof of Lemma 4.3.

Remark 3.6. The fact that $\mathrm{Sec}^{k}-\mathrm{Sec}^{k-1}$ is smooth (which follows from part (b) of Lemma 3.5) is a special case of [Bertram], Corollary 1.6.

Remark 3.7. By construction the fiber of $\mathcal{E}^{\perp}$ over a $k$-tuple of points in $\mathbb{P}^{k}=X^{(k)}$ is the $k$-plane in $\operatorname{Sym}^{n}\left(W^{*}\right)$ passing through these $k$ points and the point 0 (if the points in the $k$-tuple are not distinct, the plane becomes an osculating plane to $X$ ). Hence, the projectivization of the bundle $\mathcal{E}^{\perp}$ is the $k$ th "secant bundle" of the curve $X$ described in [Bertram], Section 1.

Let $\rho$ be a partition of $k$. The fiber bundle $p_{\mathcal{E}^{\perp}} \circ\left(\pi^{\perp}\right)^{-1}: U_{k} \rightarrow \operatorname{Conf}_{k}(X)$ yields a map of groups $\pi_{1}\left(U_{k}\right) \rightarrow \pi_{1}\left(\operatorname{Conf}_{k}(X)\right)=B_{k}\left(\mathbb{P}^{1}\right) \rightarrow S_{k}$. This allows us to make the following

Definition 3.8. The local system $\mathcal{R}_{\rho}$ on $U_{k}$ is defined by lifting the representation $\rho$ via the above map $\pi_{1}\left(U_{k}\right) \rightarrow S_{k}$. We define a perverse sheaf $\mathbf{R}_{\rho}$ as the intermediate extension of $\mathcal{R}_{\rho}$ to $\operatorname{Sym}^{n}\left(W^{*}\right)$.

Now we are ready to prove Theorem 1.1:

Proof of Theorem 1.1. We prove the statement by decreasing induction on the length of the first row of $\Lambda$ (or, equivalently, by increasing induction on $|\lambda|$ ). The base of induction is furnished by Example 1.2, where the length of the first row of $\Lambda$ is $|\Lambda|-1$.

Proposition 3.4 shows that $\Phi\left(\mathbf{L}_{\bar{\Lambda}}\right)=\pi_{*}^{\perp} p_{\mathcal{E}^{\perp}}^{*} \widetilde{L}_{\lambda}[|\lambda|]$. By Pieri's rule the irreducible decomposition of the induced representation $\bar{\Lambda}=\operatorname{Ind}_{S_{k} \times S_{n-k}}^{S_{n}}(\lambda \otimes I)$ of $S_{n}$ has exactly one irreducible representation with the first row of length at most $n-k$, this representation is $\Lambda$, and it has multiplicity 1 . Hence, $\mathbf{L}_{\bar{\Lambda}}=\mathbf{L}_{\Lambda} \oplus \bigoplus_{\Omega,|\omega|<k} a_{\Omega} \mathbf{L}_{\Omega}$, where $a_{\Omega}$ is the multiplicity of $\Omega$ in $\bar{\Lambda}$. The inductive hypothesis implies that for all $\Omega$ with $|\omega|<k$, we have $\Phi\left(\mathbf{L}_{\Omega}\right)=\mathbf{R}_{\omega}$. Hence, $\Phi\left(\mathbf{L}_{\bar{\Lambda}}\right)=\Phi\left(\mathbf{L}_{\Lambda}\right) \oplus \bigoplus_{\Omega,|\omega|<k} a_{\Omega} \mathbf{R}_{\omega}$. Since by definition $\mathbf{R}_{\omega}$ is an irreducible perverse sheaf supported on the cone over $\operatorname{Sec}^{|\omega|} \subset \operatorname{Sec}^{k-1}$, the restriction of $\Phi\left(\mathbf{L}_{\Lambda}\right)$ to $U_{k}$ coincides with the restriction to $U_{k}$ of $\Phi\left(\mathbf{L}_{\bar{\Lambda}}\right)=\pi_{*}^{\perp} p_{\mathcal{E} \perp}^{*} \widetilde{L}_{\lambda}[|\lambda|]$, which by definition is $\mathcal{R}_{\lambda}$. Note that by Lemma 3.5 (a) $\pi_{*}^{\perp} p_{\mathcal{E}^{\perp}}^{*} \widetilde{L}_{\lambda}[|\lambda|]$ (and hence $\Phi\left(\mathbf{L}_{\Lambda}\right)$ ) is supported on $\mathrm{Sec}^{k}$. Since Fourier transform of an irreducible perverse sheaf is an irreducible perverse sheaf, by the structure theorem of $[\mathrm{BBD}] \Phi\left(\mathbf{L}_{\Lambda}\right)$ must be the intermediate extension of $\mathcal{R}_{\lambda}$, that is $\mathbf{R}_{\lambda}$. 
The following is a corollary of the above proof:

Corollary 3.9. Even if the condition $|\lambda| \leq \frac{|\Lambda|+1}{2}$ does not hold, $\Phi\left(\mathbf{L}_{\Lambda}\right)$ is a direct summand in the semisimple perverse sheaf $\pi_{*}^{\perp} p_{\mathcal{E}^{\perp}}^{*} \widetilde{L}_{\lambda}[|\lambda|]$.

\section{Main theorem for any curve}

In this section, we prove Theorem 1.4 which generalizes Theorem 1.1, replacing the rational normal curve $X$ by an arbitrary smooth projective curve $C$ of genus $g$ embedded into $\mathbb{P}^{n}$. We start with a lemma:

Lemma 4.1. For a very ample line bundle $L$ on a smooth projective curve $C$, consider the embedding of $C$ into the projective space $\mathbb{P}\left(H^{0}(C, L)^{*}\right)$. Then the following two conditions are equivalent:

(a) Two k-secant planes of $C$ one of which contains the points $x_{1}, \ldots, x_{k} \in C$ and the other one $y_{1}, \ldots, y_{k} \in C$ (such that $x_{1}, \ldots, x_{k}, y_{1}, \ldots, y_{k}$ are all distinct) do not intersect;

(b) $h^{0}(L)-h^{0}(L(-D))=2 k$ for any effective divisor $D$ of degree $2 k$ (this condition is known as "D separates $2 k$ points").

Proof. First note that the projectivized span of all points of an effective divisor $D$ is given by $\mathbb{P}\left(\operatorname{Ker}\left(H^{0}(L)^{*} \rightarrow H^{0}(L(-D))^{*}\right)\right)$. If $D$ has degree $2 k$, the long exact sequence of cohomology associated with the short exact sequence $0 \rightarrow L(-D) \rightarrow$ $L \rightarrow L_{D} \rightarrow 0$ shows that the points of $D$ span a projectivized linear space of expected dimension $2 k-1$ if and only if $h^{0}(L)-h^{0}(L(-D))=2 k$. Finally, two $k$-secant planes in (a) intersect if and only if the projectivized linear space spanned by the points of $D=\left\{x_{1}, \ldots, x_{k}, y_{1}, \ldots, y_{k}\right\}$ has dimension less than $2 k-1$.

To prove Theorem 1.4, we generalize the constructions of the previous section. Recall that we have a smooth projective curve $C$ of genus $g$ and a line bundle $M$ of degree $n$ on $C$. We construct a vector bundle $\mathcal{E}_{C}$ over $C^{(k)}$ (analogous to the bundle $\mathcal{E}$ above) as a sub-bundle of the trivial bundle $C^{(k)} \times H^{0}(C, M)$ by requiring the fiber of $\mathcal{E}_{C}$ over a point $D \in C^{(k)}$ to be given by $\operatorname{Image}\left(H^{0}(C, M(-D)) \rightarrow H^{0}(C, M)\right)$. (Note that $\mathcal{E}_{C}$ is a vector bundle for $k \leq n+1-2 g$ since then $H^{1}(C, M(-D))=0$ by Serre duality.) See the diagram below.

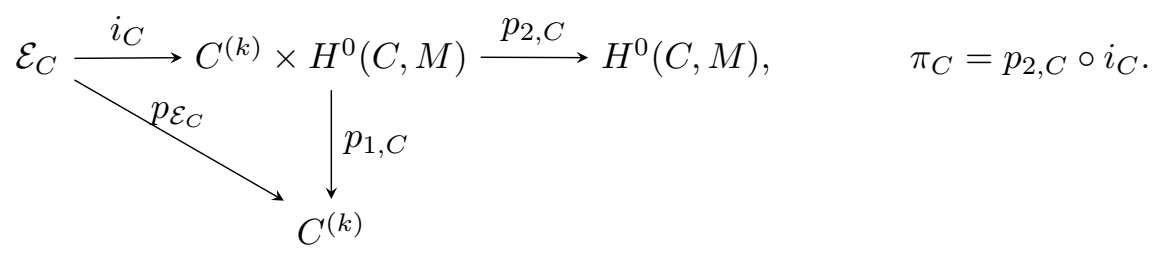

Recall that for a representation $\rho$ of $S_{r}$ we introduced the local system $\mathcal{L}_{\rho}^{C \text {,big }}$ on the open subvariety $C_{\text {dist }}^{(r)}$ of the symmetric power $C^{(r)}$. We denote its intermediate extension to $C^{(r)}$ by $L_{\rho}^{C, \text { big. }}$. We also denote the intermediate extension of the local system $\mathcal{L}_{\rho}^{C}$ to $\mathbb{P}\left(H^{0}(C, M)\right)$ by $\widetilde{L}_{\rho}^{C}$ and the intermediate extension to $H^{0}(C, M)$ of the lift of $\mathcal{L}_{\rho}^{C}$ to the cone by $\mathbf{L}_{\rho}^{C}$. The following generalizes Lemma 3.1: 
Lemma 4.2. Let $\lambda$ be a representation of $S_{k}$ and let $I$ denote the trivial representation of $S_{n-k}$. Let $\bar{\Lambda}=\operatorname{Ind}_{S_{k} \times S_{n-k}}^{S_{n}}(\lambda \otimes I)$. Then $\mathbf{L}_{\bar{\Lambda}}^{C}=\left(\pi_{C}\right)_{*}\left(p_{\mathcal{E}_{C}}\right)^{*} L_{\lambda}^{C, \text { big }}[n-k]$.

Proof. The argument parallels the proof of Lemma 3.1 with several adjustments. Namely, in place of the diagram in the proof of Lemma 3.1 we use the diagram below. In this diagram, $\phi_{r}^{C}: C^{r} \rightarrow C^{(r)}$ is the natural map for any $r, p_{1}^{\prime}$ is the first projection, and $A_{M}$ is the fiber of the Abel-Jacobi map over $M$. In addition to these, $i_{C}^{\prime}$ is the map derived from $i_{C}$ by embedding the fibers $\mathbb{P}\left(H^{0}(C, M(-D))\right)$ of $\mathbb{P}\left(\mathcal{E}_{C}\right)$ into $C^{(n-k)}$ as fibers of the Abel-Jacobi map, and $p_{\mathcal{E}_{C}}^{\prime}$ and $\pi_{C}^{\prime}$ are the analogs of $p_{\mathcal{E}_{C}}$ and $\pi_{C}$ for the projectivization $\mathbb{P}\left(\mathcal{E}_{C}\right)$ of the bundle $\mathcal{E}_{C}$.

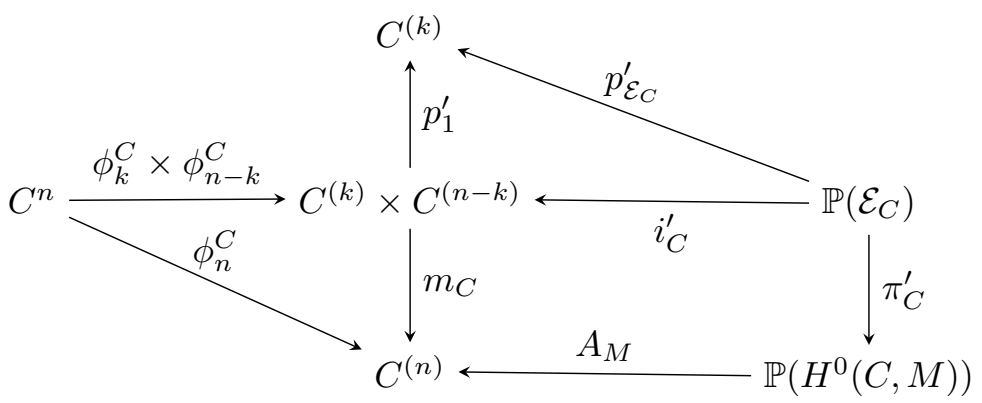

The argument of Lemma 3.1 gives $L_{\bar{\Lambda}}^{C \text {,big }}=\left(m_{C}\right)_{*}\left(L_{\lambda}^{C \text {, big }} \otimes \underline{\mathbb{C}}[n-k]\right)$. Hence,

$$
\widetilde{L}_{\bar{\Lambda}}^{C}=A_{M}^{*} L_{\bar{\Lambda}}^{C, \text { big }}=\left(\pi_{C}^{\prime}\right)_{*}\left(i_{C}^{\prime}\right)^{*}\left(L_{\lambda}^{C, \text { big }} \otimes \underline{\mathbb{C}}[n-k]\right)=\left(\pi_{C}^{\prime}\right)_{*}\left(p_{\mathcal{E}_{C}}^{\prime}\right)^{*} L_{\lambda}^{C, \text { big }}[n-k] .
$$

From this the statement of the lemma follows. (Note that we used proper base change in the second equality above.)

We also have to prove a generalization of Lemma 3.5:

Lemma 4.3. (a) The image of $\mathcal{E}_{C}^{\perp}$ under $\pi_{C}^{\perp}$ is the cone over $\operatorname{Sec}^{k}(C)$ in $H^{0}(C, M)^{*}$. (b) If $k \leq\left\lfloor\frac{n+1}{2}\right\rfloor-g$, the map $\pi_{C}^{\perp}$ is an isomorphism restricted to $\left(\pi_{C}^{\perp}\right)^{-1}\left(U_{k}^{C}\right)$ where $U_{k}^{C}$ is the cone (with vertex removed) over $\operatorname{Sec}^{k}(C)-\operatorname{Sec}^{k-1}(C)$ in $H^{0}(C, M)^{*}$.

(c) If $k \leq\left\lfloor\frac{n+1}{2}\right\rfloor-g$, the map $p_{\mathcal{E}_{C}^{\perp}}$ restricted to $\left(\pi_{C}^{\perp}\right)^{-1}\left(U_{k}^{C}\right)$ is a fiber bundle over $C_{\mathrm{dist}}^{(k)}$.

Proof. To prove (a), we note that the fiber of $\mathcal{E}_{C}$ over $D \in C^{(k)}$ is given by Image $\left(H^{0}\right.$ $\left.(M(-D)) \rightarrow H^{0}(M)\right)$, which is orthogonal to the cone over the $k$-secant plane passing through the points of $D$ given by $\operatorname{Ker}\left(H^{0}(M)^{*} \rightarrow H^{0}(M(-D))^{*}\right)$.

Parts (b) and (c) will follow if we show that the condition of part (a) of Lemma 4.1 holds. By Lemma 4.1 it is enough to show that part (b) of Lemma 4.1 holds for $k=\left\lfloor\frac{n+1}{2}\right\rfloor-g$. That is, we would like to show that $h^{0}(C, M)=h^{0}(C, M(-D))+2 k$ holds for any effective $D$ of degree $2 k$.

By Riemann-Roch we have $h^{0}(C, M)-h^{1}(C, M)=n+1-g$ and $h^{0}(C, M(-D))-$ $h^{1}(C, M(-D))=n-2 k+1-g$ for an effective $D$ with $|D|=2 k$. We have $h^{1}(M)=$ $h^{0}\left(K \otimes M^{-1}\right)$. We also have $\operatorname{deg} K=2 g-2$ so $\operatorname{deg}\left(K \otimes M^{-1}\right)=2 g-2-n<0$ because we assumed that $n>2 g-2$. Hence $h^{1}(C, M)=0$ and $h^{0}(C, M)=n+1-g$. Subtracting, we get $h^{0}(C, M)-h^{0}(C, M(-D))=h^{1}(C, M(-D))+2 k$. So to ensure that the condition of Lemma $4.1(a)$ holds it is enough to have $h^{1}(M(-D))=0$ for all 
$D$ of degree $2 k$. In other words, it is enough to make sure that $h^{0}\left(K \otimes M^{-1}(D)\right)=0$ for all $D$ of degree $2 k$. For this it is enough to have $\operatorname{deg}\left(K \otimes M^{-1}(D)\right)=0$, that is $2 g-2-n+2 k<0$. The last inequality holds for $k=\left\lfloor\frac{n+1}{2}\right\rfloor-g$.

Using Lemmas 4.2 and 4.3 in place of Lemmas 3.1 and 3.5, the proof of Theorem 1.4 is identical to the proof of Theorem 1.1.

\section{Relation with M. Brion's result}

Recall that $\otimes$ stands for the left derived tensor product of objects of $D_{c}^{b}(Y)$, where $Y$ is an algebraic variety. The following lemma will be used in this section:

Lemma 5.1. Let $f: Y \rightarrow Z$ be a finite morphism with $Y, Z$ smooth varieties of dimension $n$. Then $f_{*}(\underline{\mathbb{C}}[n]) \otimes f_{*}(\underline{\mathbb{C}}[n])$ is a perverse sheaf on $Z$.

Proof. Using base change we can write $f_{*}(\underline{\mathbb{C}}[n]) \otimes f_{*}(\underline{\mathbb{C}}[n])=g_{*}(\underline{\mathbb{C}}[n])$ where $g$ : $Y \times{ }_{Z} Y \rightarrow Z$.

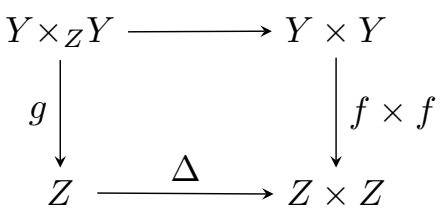

Note that $Y \times_{Z} Y$ is locally a complete intersection because locally $Y \cong \mathbb{C}^{n}$ and $Z \cong \mathbb{C}^{n}$ and $f=\left(f_{1}, \ldots, f_{n}\right): \mathbb{C}^{n} \rightarrow \mathbb{C}^{n}$ and hence $Y \times{ }_{Z} Y \subset Y \times Y$ is of dimension $n$ and given by $n$ equations $f_{i}\left(x^{\prime}\right)=f_{i}\left(x^{\prime \prime}\right)$ where $\left(x^{\prime}, x^{\prime \prime}\right) \in Y \times Y$. By a theorem of Hamm and Le [HL], Corrollary 3.2.2, the constant sheaf on a locally complete intersection is perverse; hence $\underline{\mathbb{C}}[n] \in D_{c}^{b}\left(Y \times{ }_{Z} Y\right)$ is perverse. Since $g$ is a finite map, $g_{*}(\mathbb{C}[n])$ is also perverse.

From Lemma 5.1 we deduce the following

Corollary 5.2. Let $\Lambda, \Omega$, and $\Sigma$ be partitions of $n$ and let $V=\operatorname{Sym}^{n}(W)$. Then

(a) The object $\mathbf{L}_{\Lambda} \otimes \mathbf{L}_{\Omega} \in D_{c}^{b}(V)$ is a perverse sheaf.

(b) The multiplicity of the subquotient $\mathbf{L}_{\Sigma}$ in $\mathbf{L}_{\Lambda} \otimes \mathbf{L}_{\Omega}$ is $k_{\Lambda, \Omega}^{\Sigma}$.

Proof. To prove (a), recall from the proof of Lemma 3.1 the map $\phi=\phi_{n}$ and the fact that $\widetilde{L}_{\rho}$ is a direct summand in $\phi_{*}(\underline{\mathbb{C}}[n])$ for a representation $\rho$ of $S_{n}$. Hence, $\widetilde{L}_{\Lambda} \otimes \widetilde{L}_{\Omega}$ is a direct summand in $\phi_{*}(\underline{\mathbb{C}}[n]) \otimes \phi_{*}(\underline{\mathbb{C}}[n])$ which is a perverse sheaf by Lemma 5.1 . Hence, $\widetilde{L}_{\Lambda} \otimes \widetilde{L}_{\Omega}$ is a perverse sheaf. It follows that the same is true for $\mathbf{L}_{\Lambda} \otimes \mathbf{L}_{\Omega}$. Part (b) follows by the classification theorem of perverse sheaves from the fact that on the open subvariety $\mathbb{P}^{n}-D_{n}$ we have $\mathcal{L}_{\Lambda} \otimes \mathcal{L}_{\Omega}=\mathcal{L}_{\Lambda \otimes \Omega}$.

To prove Proposition 1.5, we will use the interaction of the Fourier transform with convolution, which is defined as follows:

Definition 5.3. Let $V$ be a vector space and $s: V \times V \rightarrow V$ be the sum map and let $\mathcal{F}, \mathcal{G} \in D_{c}^{b}(V)$. Then the convolution of $\mathcal{F}$ and $\mathcal{G}$ is the object of $D_{c}^{b}(V)$ given by $\mathcal{F} * \mathcal{G}=s !(\mathcal{F} \otimes \mathcal{G})$.

Remark 5.4. The Fourier-Deligne transform sends the (derived) tensor product of $\mathcal{F}, \mathcal{G} \in D_{c}^{b}(V)$ to the convolution $\mathcal{F} * \mathcal{G}$. 
Remark 5.5. Let $\mathcal{F}, \mathcal{G} \in D_{c}^{b}(V)$ be two objects such that $\mathcal{F}$ is supported on a closed subvariety $Y$ of $V$ and $\mathcal{G}$ is supported on a closed subvariety $Z$ of $V$. Then the convolution $\mathcal{F} * \mathcal{G}$ is supported on the join $J(Y, Z)$ of $Y$ and $Z$ (where $J(Y, Z)$ is defined as the closure of the union of lines that pass through $y, z$ where $y \in Y, z \in Z$ and $y \neq z)$.

We finish with the proof of a part of M. Brion's result.

Proof of Proposition 1.5. To prove part (a), assume on the contrary that the Kronecker coefficient $k_{\Lambda, \Omega}^{\Sigma}$ is nonzero. Then by Corollary 5.2 the perverse sheaf $\mathbf{L}_{\Lambda} \otimes \mathbf{L}_{\Omega}$ has a subquotient isomorphic to $\mathbf{L}_{\Sigma}$. By exactness of the Fourier transform, it follows that the perverse sheaf $\mathbf{R}_{\lambda} * \mathbf{R}_{\omega}$ has a subquotient isomorphic to $\mathbf{R}_{\sigma}$; hence its restriction to an open subset of Sec ${ }^{|\sigma|}$ is a nonzero local system. However, by Remark 5.5 the convolution $\mathbf{R}_{\lambda} * \mathbf{R}_{\omega}$ is supported on the join of $\operatorname{Sec}^{|\lambda|}$ and $\operatorname{Sec}^{|\omega|}$ which is $\operatorname{Sec}^{|\lambda|+|\omega|}$, a closed subset of $\mathrm{Sec}^{|\sigma|}$ - a contradiction.

To prove (b), we use the diagram below.

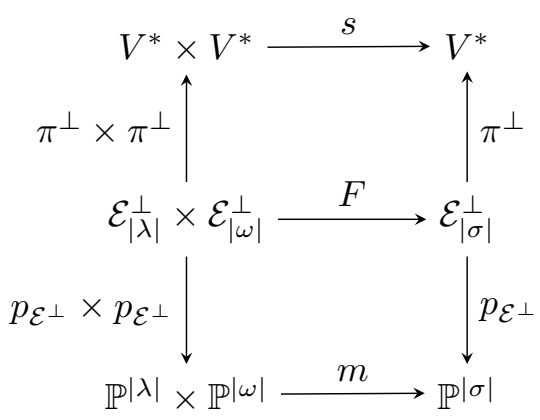

In this diagram, $\mathcal{E}_{k}^{\perp}$ is the bundle introduced in Section 3 for a specific value of $k$, $s$ is the sum map on $V^{*}=\operatorname{Sym}^{n}\left(W^{*}\right), m$ is the map from the proof of Lemma 3.1, and $F$ is the map which is the product of polynomials on the base of $\mathcal{E}^{\perp}$ 's and the sum in the fibers of $\mathcal{E}^{\perp}$ 's. It is clear that $F$ is well defined and the diagram commutes.

By the proof of Lemma 3.1, $m_{!}\left(\widetilde{L}_{\lambda} \otimes \widetilde{L}_{\omega}\right)=\bigoplus_{\sigma,|\sigma|=|\lambda|+|\omega|} c_{\lambda, \omega}^{\sigma} \widetilde{L}_{\sigma}$. By proper base change in the diagram above we see that

$$
s_{!}\left(\pi_{*}^{\perp} p_{\mathcal{E}^{\perp}}^{*} \widetilde{L}_{\lambda}[|\lambda|] \otimes \pi_{*}^{\perp} p_{\mathcal{E}^{\perp}}^{*} \widetilde{L}_{\omega}[|\omega|]\right)=\bigoplus_{\sigma,|\sigma|=|\lambda|+|\omega|} c_{\lambda, \omega}^{\sigma}\left(\pi_{*}^{\perp} p_{\mathcal{E}^{\perp}}^{*} \widetilde{L}_{\sigma}[|\sigma|]\right) .
$$

By the proof of Theorem 1.1, $\pi_{*}^{\perp} p_{\mathcal{E} \perp}^{*} \widetilde{L}_{\mu}[|\mu|]$ coincides with $\mathbf{R}_{\mu}$ on an open dense subvariety of Sec ${ }^{|\mu|}$; therefore $\left(\pi_{*}^{\perp} p_{\mathcal{E}{ }^{\perp}}^{*} \widetilde{L}_{\lambda}[|\lambda|]\right) *\left(\pi_{*}^{\perp} p_{\mathcal{E}{ }^{\perp}}^{*} \widetilde{L}_{\omega}[|\omega|]\right)$ coincides with $\mathbf{R}_{\lambda} * \mathbf{R}_{\omega}$ on an open dense subvariety of $\operatorname{Sec}^{|\lambda|+|\omega|}$.

By Corollary 5.2, the tensor product $\mathbf{L}_{\Lambda} \otimes \mathbf{L}_{\Omega}$ is a perverse sheaf so its Fourier transform $\mathbf{R}_{\lambda} * \mathbf{R}_{\omega}$ is perverse as well. Above we detected all the subquotients in $\mathbf{R}_{\lambda} * \mathbf{R}_{\omega}$ whose support is of maximum dimension $|\lambda|+|\omega|$; by the classification of perverse sheaves, they are the same as the subquotients with support of maximum dimension in $\bigoplus_{\sigma,|\sigma|=|\lambda|+|\omega|} c_{\lambda, \omega}^{\sigma}\left(\pi_{*} p_{\mathcal{E} \perp}^{*} \widetilde{L}_{\sigma}[|\sigma|]\right)$. By the proof of Theorem 1.1, all these subquotients are of the form $\mathbf{R}_{\sigma}$, where $|\sigma|=|\lambda|+|\omega|$ and each subquotient $\mathbf{R}_{\sigma}$ occurs $c_{\lambda, \omega}^{\sigma}$ times. By exactness of the Fourier transform, $c_{\lambda, \omega}^{\sigma}$ must be equal to the multiplicity of the subquotient $\mathbf{L}_{\Sigma}$ in $\mathbf{L}_{\Lambda} \otimes \mathbf{L}_{\Omega}$, which is $k_{\Lambda, \Omega}^{\Sigma}$ by Corollary 5.2. 


\section{Acknowledgments}

The author is very grateful to Roman Bezrukavnikov for suggesting this problem and for many useful discussions and ideas. The author is grateful to J.M. Landsberg for helping her locate the reference for M. Brion's result. She also thanks A. Beilinson, S. Bloch, A. Libgober, L.-H. Lim, M. Mella, L. Oeding, R. Piene, and B. Sturmfels for useful conversations.

\section{References}

[BBD] A.A. Beilinson, J. Bernstein and P. Deligne, Faisceaux pervers, Asterisque 100, (1982).

[Bertram] A. Bertram, Moduli of rank-2 vector bundles, theta divisors, and the geometry of curves in projective space, J. Diff. Geom. 35 (1992), 429-469.

[Brion] M. Brion, Stable properties of plethysm: on two conjectures of Foulkes, Manuscripta Math. 80(4) (1993), 347-371.

[B] J.-L. Brylinski, Transformation canoniques, dualite projective, theorie de Lefschetz, transformation de Fourier et sommes trigonometriques, Asterisque 140-141 (1986), 3-134.

[BMV1] J.-L. Brylinski, B. Malgrange and J.-L. Verdier, Transformation de Fourier geometrique I, C. R. Acad. Sci. Paris Ser. I Math. 297(1) (1983), 55-58.

[BMV2] J.-L. Brylinski, B. Malgrange and J.-L. Verdier, Transformation de Fourier geometrique II, C. R. Acad. Sci. Paris Ser. I Math. 303(5) (1986), 193-198.

[CS] G. Comas and M. Seiguer, On the rank of a binary form, Found. Comput. Math. 11(1) (2011), 65-78.

[D] V.G. Drinfeld, Two dimensional $\ell$-adic representations of the fundamental group of a curve over a finite field and automorphic forms on GL(2), Amer. J. Math. 105 (1983), 85-114.

[HL] H. Hamm and D.T. Le, Rectifed homotopical depth and Grothendieck conjectures, in 'Grothendieck Festschrift II' (P. Cartier et al., eds.) Progress in Mathematics 87, Birkhauser, Boston, 1990, 311-351.

[I] L. Illusie, Deligne's $\ell$-adic Fourier transform, in 'Algebraic Geometry Bowdoin 1985' (S.J. Bloch ed.), Proc. Symp. in Pure Mathematics 46 II, American Mathematical Society, Providence, 1987, 151-163.

[KS] M. Kashiwara and P. Schapira, Sheaves on Manifolds Grundlehren der Math. Wiss. 292, Springer-Verlag, Berlin, 1990.

[KW] R. Kiehl and R. Weissauer, Weil conjectures, perverse sheaves and l'adic Fourier transform, Ergebnisse der Mathematik und ihrer Grenzgebiete. 3. Folge. 42, Springer-Verlag, Berlin, 2001.

[L] J.M. Landsberg, Tensors: geometry and applications, Graduate Studies in Mathematics 128, American Mathematical Society, Providence, 2012.

Department of Mathematics, University of Chicago, 5734 S. University Avenue, Chicago, IL 60637, USA

E-mail address: dobrovol@math.uchicago.edu 